\title{
FUNCTIONAL ECHOCARDIOGRAPHY IN THE ITALIAN NEONATAL INTENSIVE CARE UNITS: A NATIONAL SURVEY
}

I. Corsini 1*, B. Ficial2*, S. Fiocchi3 ${ }^{*}$, F, Schena ${ }^{*}$, I. Capolupo5, R.M. Cerbo6, M. Condò7, D. Doni8,

S. La Placa9, S. Porzio 10, K. Rossi1 11, S. Salvadori 12, M. Savoia ${ }^{13}$ f;

On the behalf of the Study Group of Neonatal Cardiology of the Italian Society of Neonatology.

1 Neonatal Intensive Care Unit, AOU Careggi, Florence, Italy, 2 Neonatal Intensive Care Unit, AOUI Verona, Verona, Italy, 3 Neonatal Intensive Care Unit, Niguarda, Milan, Italy, 4 Neonatal Intensive Care Unit, IRCCS Fondazione Cà Granda Ospedale Maggiore Policlinico di Milano, Milan, Italy, 5 Neonatal Intensive Care Unit, Ospedale Pediatrico Bambino Gesù, Taormina, Italy, 6 Neonatal Intensive Care Unit, Policlinico San Matteo, Pavia, Italy, 7 Neonatal Intensive Care Unit, Ospedale A. Manzoni, Lecco, Italy, 8 Neonatal Intensive Care Unit, FMBBM San Gerardo, Monza, Italy, 9 Neonatal Intensive Care Unit, AOUP Giaccone, Palermo, Italy, 10 Neonatal Section, San Michele Hospital, Naples, Italy, 11 Neonatal Intensive Care Unit, Policlinico di Modena, Modena, Italy, 12 Neonatal Intensive Care Unit, AO Padova, Padua, Italy, 13 Neonatal Intensive Care Unit, Azienda Sanitaria Universitaria Integrata di Udine, Udine, Italy

$f$ Chair of the Italian Study Group of Neonatal Cardiology of Italian Society of Neonatology

\section{Background and Aims}

Functional echocardiography (f-Echo) has increasingly been used by neonatologists to assess the hemodynamic status in neonates. Aim of this survey was to investigate the utilization of $f$-Echo in the italian Neonatal Intensive Care Units (NICUs).

\section{Method}

We conducted an on-line survey study from June to September 2017. A 37-item questionnarie was developed by the Italian Neonatal Cardiology Study Group and was sent to chiefs of italian NICUs and neonatologists with expertise in neonatal echocardiography.

\section{$\mathbf{A}$}

NEONATE IN STABLE CONDITION

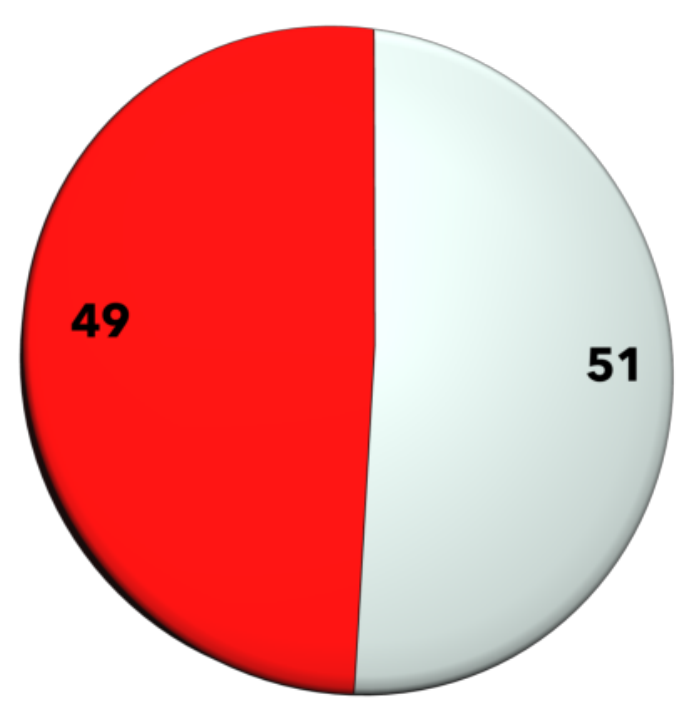

Neonatologist

Cardiologist

B

NEONATE IN CRITICAL CONDITION

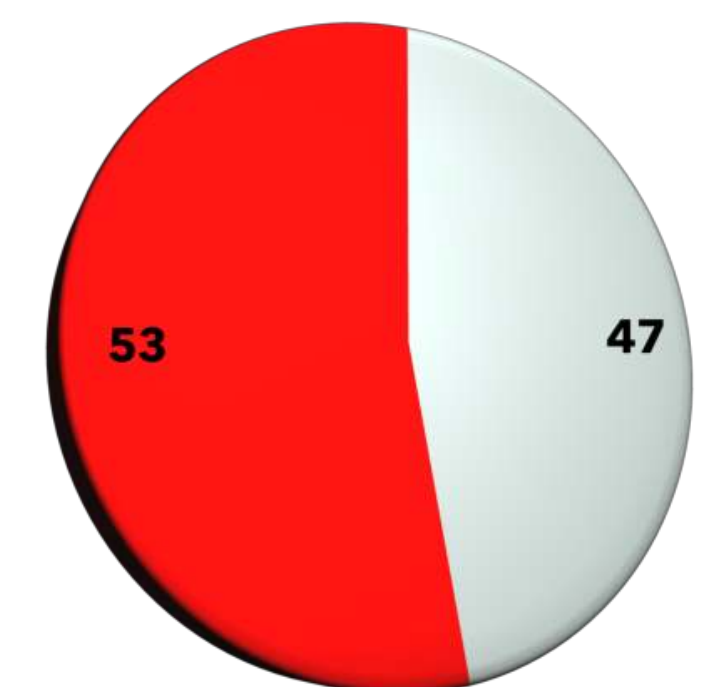

Neonatologist

Cardiologist

Practitioner performing echocardiographic assessment in order to exclude CHD in stable (A) and in critically ill patient (B)

\section{Results}

The overall survey response rate was 79\% 194 center out of 114 contacted).

In the $95 \%$ of NICUs f-Echo is used in daily clinical practice, by neonatologists, cardiologists or both $159 \%, 15 \%$ and $26 \%$ respectively). Of this centers $100 \%$ use f-echo to assess neonates with patent ductus arteriosus and persistent pulmonary hypertension of the newborn, 91\% to assess neonates with hypotension or shock, $84 \%$ to assess neonates with hypoxic-ischemic encefalopathy, $81 \%$ in case of suspicion of cardiac tamponade and $74 \%$ to assess the position of catheter's tip. Structural echocardiography to exclude congenital heart disease (CHD) in a stable neonate and in a critically ill neonate is performed exclusively by neonatologists in $51 \%$ and $47 \%$ of cases respectively.

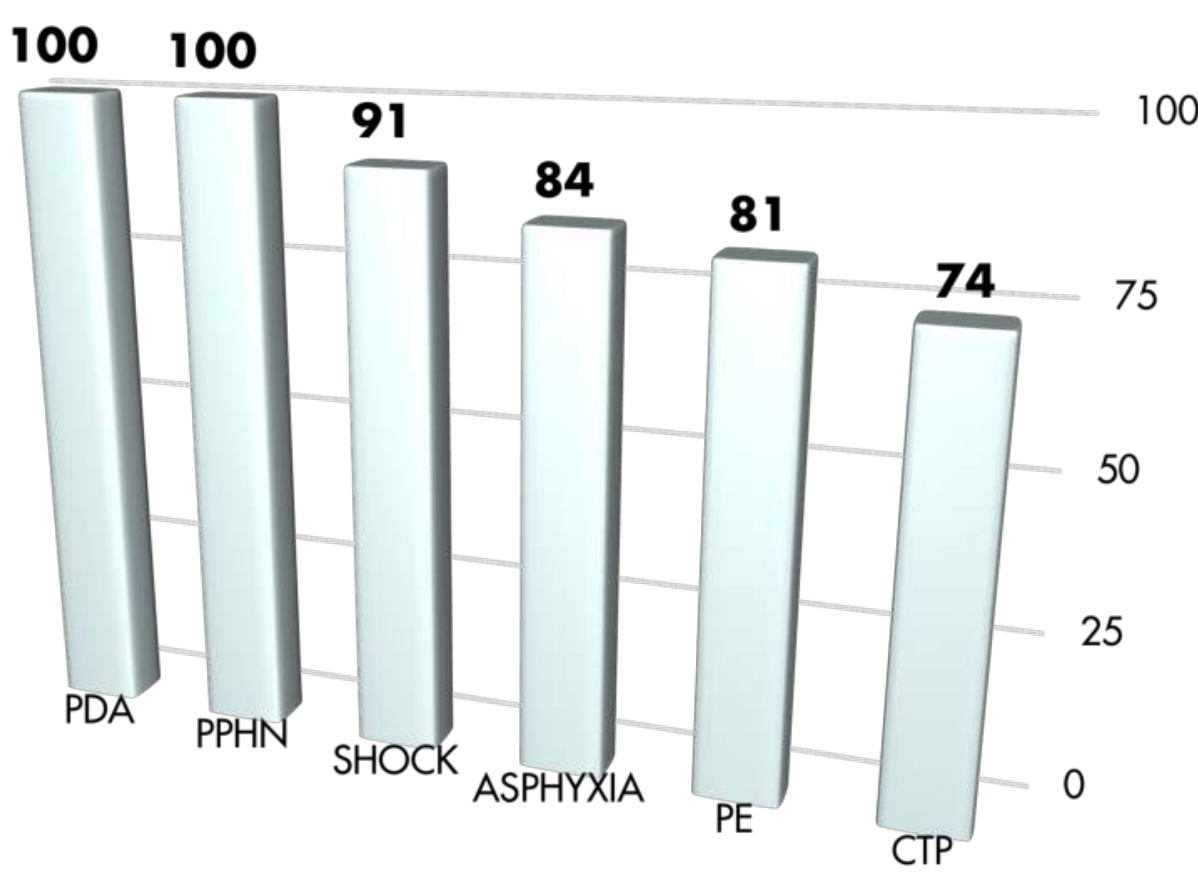

Proportion of center that use f-Echo divided for neonatal condition. PDA: Patent ductus arteriosus; PPHN: Persisten Pulmonary Hypertension of the Newborn;PE: Pericardial Effusion; CTP: Cathter Tip's Position

\section{Conclusion}

F-Echo is widely used in italian NICUs. In most centers it is performed by neonatologists.

Structural echocardiography to exclude CHD is often performed by

neonatologists. There is an urgent need of a formal training process and accreditation at a national level to standardize the use of functional echocardiography. 